\title{
Fuzzy Gain Scheduling PID Control for Position of the AR.Drone
}

\author{
Agung Prayitno ${ }^{1}$, Veronica Indrawati ${ }^{2}$, Ivan Immanuel Trusulaw ${ }^{3}$ \\ Department of Electrical Engineering, University of Surabaya (UBAYA), Indonesia
}

\begin{tabular}{l}
\hline \hline Article Info \\
\hline Article history: \\
Received May 31, 2017 \\
Revised Oct 24, 2017 \\
Accepted Mar 14, 2018 \\
\hline
\end{tabular}

Keyword:

AR.Drone

Fuzzy gain scheduling

PID control

Position control

Takagi-sugeno-kang fuzzy

\begin{abstract}
This paper describes the design and implementation of fuzzy gain scheduling PID control for position of the AR.Drone. This control scheme uses 3 PID controllers as the main controller of the AR.Drone, in this case to control pitch, roll and throttle. The process of tuning parameters for each PID is done automatically by scheduling determined by Takagi-Sugeno-Kang (TSK) fuzzy logic model. This paper uses five function sets of PID parameters that will be evaluated by fuzzy logic in order to tune PID controllers. Error position $(\mathrm{x}, \mathrm{y}, \mathrm{z})$, as inputs of controller, enters the PID Signal block yielding the ouputs in term of error, integral error and differential error. These signal become the inputs of the fuzzy scheduler to yield outputs pitch, roll and throttle to the AR.drone. The control scheme is implemented on the AR.Drone to make it fly to forming a square in the room. The experimental results show that the control scheme can follow the desired points, and process scheduling PID parameters can be shown.
\end{abstract}

Copyright $\odot 2018$ Institute of Advanced Engineering and Science. All rights reserved.

\section{Corresponding Author:}

Agung Prayitno,

Department of Electrical Engineering,

University of Surabaya (UBAYA),

TB.2 Building, Raya Kalirungkut 60293 Surabaya, East Java, Indonesia.

Email: prayitno_agung@staff.ubaya.ac.id

\section{INTRODUCTION}

The use of quadrotor to assist human activities has become more diverse. The examples are the use of quadrotor for news coverage activities, assisting agriculture, disaster evacuation, product promotion and entertainment. Research using the quadrotor is also increasingly masive in various universities in the world both in terms of hardware and software. Many quadrotor platforms for research purposes are sold on the market with a range of low to expensive prices with specific technical specifications.

This research uses the AR.Drone 2.0 Elite Edition which is a product of Parrot. The AR.Drone is relatively inexpensive and is equipped with onboard electronics that have a motherboard, a processor, a Wi-Fi chip, 3 axis gyroscope, 3 axis accelerometer, a sonar altimeter, and a front camera and bottom. It is also equipped with a real-time operating system that can perform multiple tasks simultaneously, such as communication with a PC via Wi-Fi, video data sampling, sensor acquisition, image processing, state estimation and closed loop control. With this communication, it is possible to transmit control commands and request navigation data of drones in the form of actual roll value, sideward speed, actual pitch value, forward speed, actual yaw rate value, yaw value, vertical rate value and altitude value [1]. Maneuver of the AR.Drone can be controlled with 4 pieces of control command that pitch, roll, yaw and throttle that has values between -1 and 1 as shown in Table 1 .

Until now, many studies have been using the AR.Drone as a platform. Mogenson [2] designed the AR.Drone LabVIEW toolkits that facilitate lecturers and researchers to control the AR.Drone using their controller design. There are 4 pieces of soft Virtual Instrument (VI), namely Main VI, Video VI, NavData VI and State VI. Main VI transmits control commands and keeps the communication channel running. Video VI is used to read UDP that contain video frame packets sent from the AR.Drone, turn it into an image or pixel 
clusters. NavData VI sends UDP packets to the Navdata output who ordered AR.Drone to send sensor's data to IP address of the computer.

Table 1. Control Command of AR.Drone

\begin{tabular}{ccc}
\hline Input & Positive values & Negative values \\
\hline Pitch & Backward & Forward \\
Roll & Right & Left \\
Yaw & Rotate CW & Rotate CCW \\
Throttle & Up & Down \\
\hline
\end{tabular}

State VI is used to estimate $\mathrm{x}, \mathrm{y}, \mathrm{z}$ position of the Navdata. Some researchers noted having designed PID and Fuzzy controller on the AR.Drone. Prayitno, et al. in [3] designed 2 fuzzy controllers to control the $\mathrm{x}$ and y position using pitch and yaw. Indrawati, et al. in [4] and [5] is designed and implemented three pieces of fuzzy logic control to control the position of $x, y, z$ using of pitch, roll and vertical rate of the AR.Drone. They compared various schemes of fuzzy control to position control. Prayitno, et al. in [6] is designed conventional PID control and compared with fuzzy control scheme designed by Indrawati in [5]. Tang in [7] designed a PID controller for waypoint navigation applications and trajectory tracking and vision-based controller for a variety of formation flying. Abbas, et al. in [8] is designed controls for tracking formation quadrotor where the PID controller is implemented in quadrotor leader and directed Lyapunov controller is implemented on the followers. The artificial fish swarm algorithm is used for dynamics optimization of the parameters controllers. Seidabad, et al. in [9] modelled the motion of all quadrotor with Simulink. They are using two types of controllers, which are PID controller and combination of fuzzy-PID controller. The simulation results showed that the hybrid fuzzy-PID controller is more suitable when there has a turbulence. Gautam, et al. in [10] designed a self-tuning PID controller using EKF algorithm that is implemented on quadrotor for attitude and position control of the quadrotor. Ammozgar, et al. in [11] implemented Fuzzy PID Gain-Scheduled to cope with the possible failure of the actuator quadrotor. The two actuator failure schemes are designed, which are the failure of all actuators and single actuator. Prayitno, et al. in [12] implemented other control scheme, H-Infinity, to control pitch and roll of the AR.Drone. Hazzabi, et al. in [13] implemented adaptive FLC-PI, where PI controller parameters are adjusted by fuzzy gain scheduling, to control an induction motor. Syed, et al. in [14] applied fuzzy gain scheduling PI control based on the system's operating conditions for controlling engine power and speed of a power-split HEV in the applied automotive field.

Our paper describes the design and implementation the fuzzy gain scheduling PID control for position of the AR.Drone based on operating conditions. This research is motivated by the fact that in nonlinear system, a single set of PID gain will only be suitable for a given operating point. When the aircraft, in this case the AR.Drone, fly on a different operating point, it would require a different PID gain. In order to make the drone fly to multiple operating points, multiple sets of PID gain is required. The scheduling mechanism is needed in order to determine the PID gain which is appropriate for the current operating point. Once the new operating point is detected, the PID gains can be changed to the appropriate values. The gain scheduler consists of multiple sets of PID gain and the logic for detecting the operating point and choosing the corresponding value of PID Gain. The purpose of this research is to experiment the implementation of fuzzy gain scheduling PID control for position control of AR.Drone in the laboratory. In this research, as the gain scheduler, Takagi-Sugeno-Kang (TSK) fuzzy logic is used. The suitable PID gain function for some operating point is determined by experimentally to get the expected transient response. Five functions of PID gain represent five operating point are used in this research. This research shows that the gain scheduling pid control is successfully applied to AR.Drone position control.

\section{RESEARCH METHOD}

\subsection{Basic setup of AR.Drone}

This research uses LabVIEW software by modifying the program created by [2]. This program uses communication and basic control for AR.Drone by utilizing 2 main Virtual Instrument (VI) that is AR Drone Poly Main Fly and AR Drone Read Nav Data. AR Drone Poly Main Fly to send flying commands while AR Drone Read Nav Data to receive navigation data of the AR.Drone. In AR Drone Poly Main Fly there are 4 states, namely SETUP, ATREF, ATPCMD, and WATCHDOG.

a. State SETUP is used to send the first set of data needed before AR.Drone starts flying. The data contained the desired AR.Drone flying specification consisting of Drone Command Port, Drone IP Address, Home Command Port, Max Altitude (m), Max Pitch and Roll Angle (deg), Max Vertical Speed (m/s), Max Yaw 
Speed (deg/s), Flight Mode, Mounted Hull, and Video Camera. The data transmission ends with an AT*FTRIM command which is useful for determining the reference slope of the ground. AR.Drone will only be ready to fly when placed on a horizontal plane.

b. State ATREF sends commands related to the flying conditions of AR.Drone, such as Fly, Land and Emergency Land. The command that is sent has the format $A T^{*} R E F=$ [Sequence], [Argument] $\langle C R\rangle$. The sequence is the sequence number of the command, AR.Drone simply executes a command that has a sequence number larger than the previous sequence number so AR.Drone does not re-do the previous command. The program used in this study uses sequence numbers up to 100 and will be reset to 1 when it reaches 100. The argument is a 32-bit integer with each bit having its own function. Bits 0 through 7 are not used and have a value of 0 . Bit 8 is used to set the emergency land mode by giving a value of 1 . When bit 8 is 0 then AR.Drone is in normal mode and ready to accept the command. Emergency land mode is a condition where AR.Drone will turn off the entire motor without regard to the given command. Bit 9 is used to set the fly or land mode of AR.Drone. When bit 9 is set 1 then AR.Drone is ordered to fly and vice versa when set 0 then AR.Drone will make a landing. Bits 10 through 31 are not used and have a fixed value of "000000001010101010".

c. State ATPCMD is useful for sending commands for AR.Drone to move or maneuver with pitch, roll, yaw and throttle. The format of the command is AT * PCMD = [Sequence], [Flag], [Roll], [Pitch], [Throttle], [Yaw] $\langle\mathrm{CR}\rangle$. The sequence is the sequence number of the command, AR.Drone simply executes a command that has a sequence number larger than the previous sequence number so AR.Drone does not re-do the previous command. The program used in this study uses sequence numbers up to 100 and will be reset to 1 when it reaches 100 . Bit 0 is used to select pitch, roll, and yaw commands sent to AR.Drone or just hover in place. Changing the bit value to 0 means AR.Drone is in hover mode, and changing its value to 1 means AR.Drone can perform translational and rotational movements in the fields of $\mathrm{X}$ and $\mathrm{Y}$. Bit 1 is used to instruct AR.Drone to process roll, pitch, yaw, and throttle arguments filled with the desired values for AR.Drone maneuvers. The minimum value for each argument is -1 , with a maximum value of 1 . These values need to be converted first into hexadecimal numbers and converted back to 32-bit decimal numbers.

d. State WATCHDOG is a state assigned to send commands of AT*COMWDG. The command does not require any arguments, as it only serves to keep the connection between the computer and AR.Drone. AT * COMWDG commands must be sent at least every $300 \mathrm{~ms}(0.3$ seconds $)$. If AR.Drone does not receive the command within a period of more than $300 \mathrm{~ms}$, then the commands entered into AR.Drone will be ignored because AR.Drone assumes that the connection between AR.Drone and the controller is being disconnected. The program created for this study of WATCHDOG states is sent every $150 \mathrm{~ms}$, assuming that if the sending command is interrupted and $\mathrm{AT} * \mathrm{COMWDG}$ is not received by AR.Drone then sending commands at subsequent intervals (300 ms) may prevent disruption of connections between computers and AR.Drone.

\subsection{The fuzzy gain scheduling PID control}

The fuzzy gain scheduling PID control system scheme is implemented on the AR Drone is shown in Figure 1. The movement of drone to the position $\mathrm{x}, \mathrm{y}$ and $\mathrm{z}$ are controlled by 3 controllers; each controls the pitch, roll and vertical rate. The PID controllers are used as the main controller. While fuzzy is used as the scheduler of the gain parameters of PID corresponding to earn operating point of drones, evaluated on the error position. Each fuzzy gain scheduling PID block has the same scheme. Figure 2 shows the Fuzzy gain scheduling PID for $\mathrm{x}$ position. For $\mathrm{y}$ position and $\mathrm{z}$ position have identical scheme. This block consists of PID signals and Fuzzy scheduler. For the PID control, the general equation of the controller is used as shown (1):

$$
O(t)=K_{P} e(t)+K_{I} \int e(t) d t+K_{D} \frac{d e(t)}{d t}
$$

where:

$$
\begin{aligned}
& K_{P}: \text { Proportional Constant } \\
& K_{I}: \text { Integral Constant } \\
& K_{D}: \text { Derivative Constant }
\end{aligned}
$$

Takagi-Sugeno-Kang (TSK) model is used as a fuzzy scheduler. The rules of TSK model are shown in (2).

$$
R_{i} \text { : If } x_{i} \text { is } A_{i} \text { then } f_{i}(x), i=1,2, \ldots, n
$$


where $f_{i}(x)$ are functions.

In the fuzzy scheduler, error position, $\left(e_{x}, e_{y}, e_{z}\right)$, are used as the inputs which will be fuzzified into 5 memberships function; NB:Negative Big $(-1 \leq \mathrm{e} \leq-0.5)$, NS:Negative Small $(-0.5 \leq \mathrm{e} \leq 0)$, Z:Zero $(-0.5 \geq \mathrm{e} \leq 0.5)$, PS:Positive Small $(0 \leq \mathrm{e} \leq 0.5)$ and PB:Positive Big $(0.5 \leq \mathrm{e} \leq 1)$. Rules evaluation are defined by using (2), where $f_{i}(x)$ are PID equation in (1) based on defined operating points. The operating points are represented in five membership functions above. This research using five same rules for $x, y$ and $z$ position control as follows:

$$
\begin{aligned}
& R_{1} \text { : If } e(t) \text { is NB then } O_{1}=1.6 e(t)+0.005 \int e(t) d t+0.25 \frac{d e(t)}{d t} \\
& R_{2}: \text { If } e(t) \text { is NS then } O_{2}=0.5 e(t)+0.007 \int e(t) d t+1 \frac{d e(t)}{d t} \\
& R_{3}: \text { If } e(t) \text { is Z then } O_{3}=0 e(t)+0 \int e(t) d t+0 \frac{d e(t)}{d t} \\
& R_{4}: \text { If } e(t) \text { is PS then } O_{4}=0.5 e(t)+0.007 \int e(t) d t+1 \frac{d e(t)}{d t} \\
& R_{5}: \text { If } e(t) \text { is PS then } O_{5}=1.6 e(t)+0.005 \int e(t) d t+0.25 \frac{d e(t)}{d t}
\end{aligned}
$$

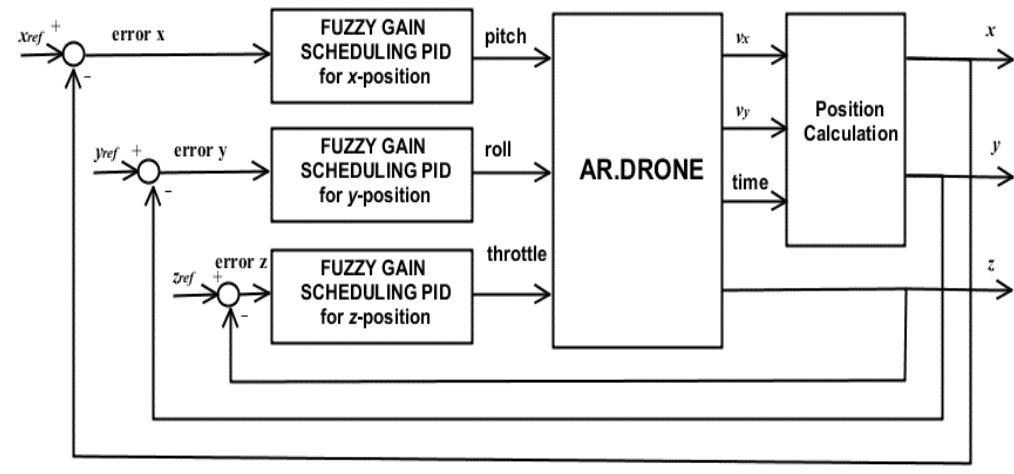

Figure 1. Block diagram fuzzy gain scheduling PID controlled system

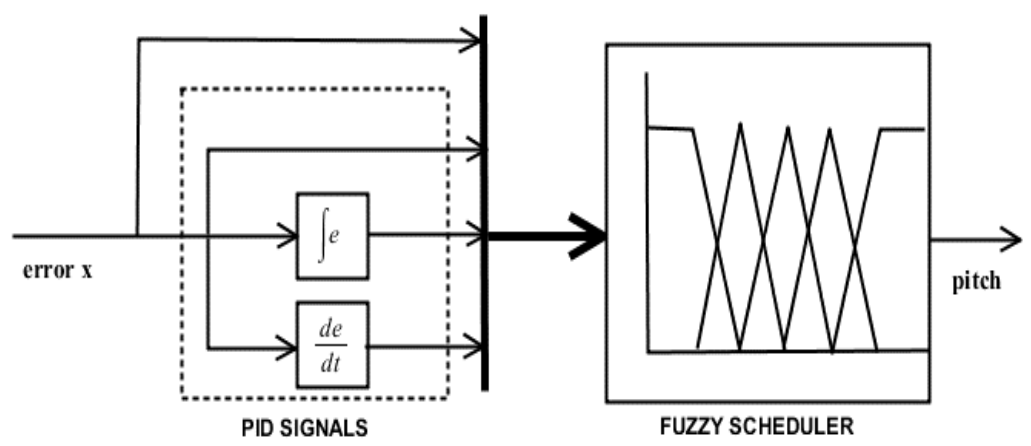

Figure 2. Fuzzy gain scheduling PID scheme for $x$-position

Each rule represents one operating point which is expressed in one set PID parameter, $\left(K_{P}, K_{I}, K_{D}\right)$, forming a PID equation. These parameters produce an expected transient response which corresponds to each operating point. The PID parameters is tuned experimentally by flying the AR.Drone to $x$ position, then analyzing the transient response. PID parameter tuning procedure for $x$ position can be written as follows, 
which is also the procedure for tuning $y$ and $z$ position. But in this research we used the same parameters for $y$ and $z$.

a. PC at the ground station has prepared a program with PID controlled system and data acquisition

b. Setpoint position selected is \pm 1 meter and \pm 0.5 meter in accordance to the defined operating points.

c. AR.Drone is flown autonomously by using P-controller setpoint 1 meter from coordinate $(0,0,1)$ to $(1,0,1)$ with a particular $K_{P}$. The data of drone is stored and the transient response is analyzed, in this case the rise time and the overshoot. It repeats 5 times. Perform this step using several $K_{P}$ and select $K_{P}$ that provide the expected transient response.

d. AR.Drone is flown autonomously using PI-controller with $K_{P}$ values obtained in step 3 and a particular $K_{I}$ from coordinate $(0,0,1)$ to $(1,0,1)$. The data of drone is stored and the transient response is analyzed, in this case the rise time and the overshoot. It repeats 5 times. Perform this step using several $K_{I}$ and select $K_{I}$ that provide the expected transient response.

e. AR.Drone is flown autonomously using PID-controller with $K_{P}$ and $K_{I}$ values obtained in step 3,4 and a particular $K_{D}$ from coordinate $(0,0,1)$ to $(1,0,1)$. The data of drone is stored and the transient response is analyzed, in this case the rise time and the overshoot. It repeats 5 times. Perform this step using several $K_{D}$ and select $K_{D}$ that provide the expected transient response

f. Perform step 3,4 and 5 with setpoint position of 0.5 meter.

g. For -1 meter position use the result from step 5, whereas for -0.5 meter position use the result of step 6. It has been confirmed by a number of attempts which resulted in relatively similar response.

h. On 0 meter position $K_{P}, K_{I}$ and $K_{D}$ is 0 (zero)

i. Tabulate the value of $K_{P}, K_{I}$ and $K_{D}$ for each position representing the membership function

The result of PID parameters gained by experimentally at each operating point can be tabulated in Table 2.

Table 2. The PID Parameters

\begin{tabular}{cccccccccc}
\hline Gain & $\begin{array}{c}\text { Operating } \\
\text { Points [m] }\end{array}$ & $K_{P}$ & $K_{I}$ & $K_{D}$ & Pitch & Roll & Throttle & $\mu$ & \multicolumn{2}{c}{ Corresponds to } \\
& -1 & 1.6 & 0.005 & 0.25 & Backward & Left & Down & NB & $R_{1}$ \\
I & -0.5 & 0.5 & 0.007 & 1 & Backward & Left & Down & NS & $R_{2}$ \\
II & 0 & 0 & 0 & 0 & Hover & Hover & Hover & Z & $R_{3}$ \\
III & 0.5 & 0.5 & 0.007 & 1 & Forward & Right & Up & PS & $R_{4}$ \\
IV & 1 & 1.6 & 0.005 & 0.25 & Forward & Right & Up & PB & $R_{5}$ \\
V & 1 & & & & & & & & \\
\hline
\end{tabular}

For $n$ rules the function can be combined to get a function, as an output of the controller, as follows

$$
R(x)=\frac{A_{1}(x) f_{1}(x)+A_{2}(x) f_{2}(x)+\cdots+A_{n}(x) f_{n}(x)}{A_{1}(x)+A_{2}(x)+\cdots+A_{n}(x)}
$$

\section{RESULTS AND ANALYSIS}

Fuzzy gain scheduling PID algorithm has been designed to be implemented on the AR.Drone 2.0 Elite Edition and tested indoor. The testing room size $6 \mathrm{~m}$ x $6 \mathrm{~m}$ x $4 \mathrm{~m}$ with a floor made of striped line for the drone use its bottom camera to estimate $x$ and $y$ position in flight. While the $z$ position using ultrasonic sensors provided onboard the drone. To test the fuzzy gain scheduling PID algorithm, AR.Drone was flown point to point toward the $\mathrm{X}$-axis from the initial coordinates $[0,0,1]$ to the coordinates $[1,0,1]$. The same test is also done using PID controller algorithm. The response of AR.Drone will be compared with the test results using PID controller. The results of both are compared and shown in Figure 3. From the test results seen that AR.Drone with PID controller tend to experience overshoot approximately $20 \%$. While AR.Drone with fuzzy gain scheduling PID gives better response with overshoot approximately $10 \%$ and settling time about 15 seconds. The next test, AR.Drone will be flown to several positions that form a grid on the coordinates $x, y, z$. The testing procedure is performed as follows:

a. Enter the reference position that will be addressed by the drones on the front panel of the software that has been made. In this test, a reference that will be addressed is $(1.5,0,1.5)$ then to $(1.5,1.5,1.5)$, and to $(0,1.5,1)$, finally back to the initial position $(0,0,1)$.

b. AR.Drone is flown manually to hover at position $(0,0,1)$. 
c. Switch ON auto, the AR.Drone will be autonomously flying toward predetermined reference. Change of set point is done if the position of the drone has been entered in error tolerances specified in the program,

d. Test is performed 5 times.

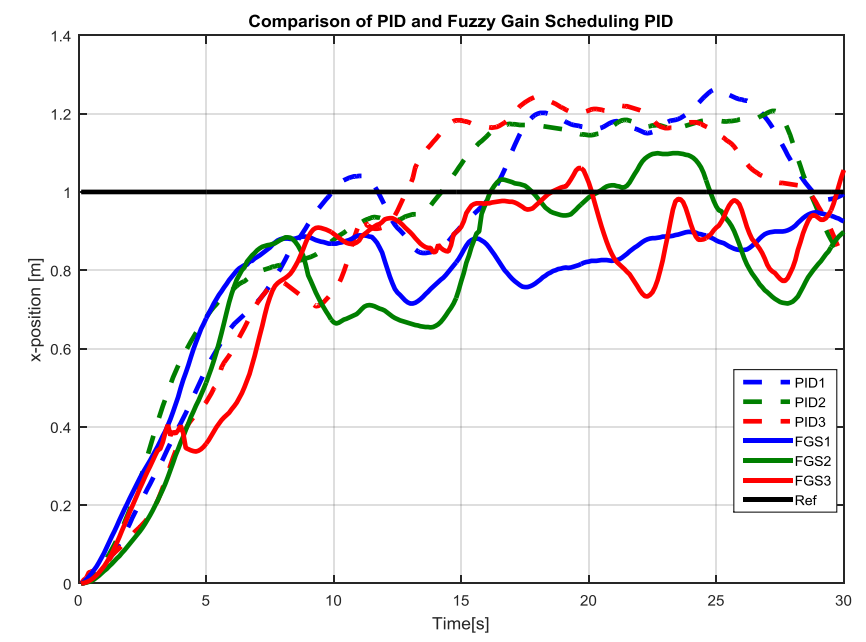

Figure 3. Comparison between PID response and Fuzzy Gain Scheduling PID response

The test result is shown in Figure 4. The picture on the left is the system response in 3 dimensions while the right image is the position response of each coordinate $\mathrm{x}, \mathrm{y}$, and $\mathrm{z}$. The depiction of 3D showed that the AR.Drone can go to any desired reference point but it has a relatively large error in the position z. There are 2 of 5 times where the experiment yields a relatively good transient response. Experiments 1 and 3 were late when switching to get to the point $(1.5,1.5,1.5)$. In general, each point can be achieved within a rise time of 10 seconds as seen in the left image.
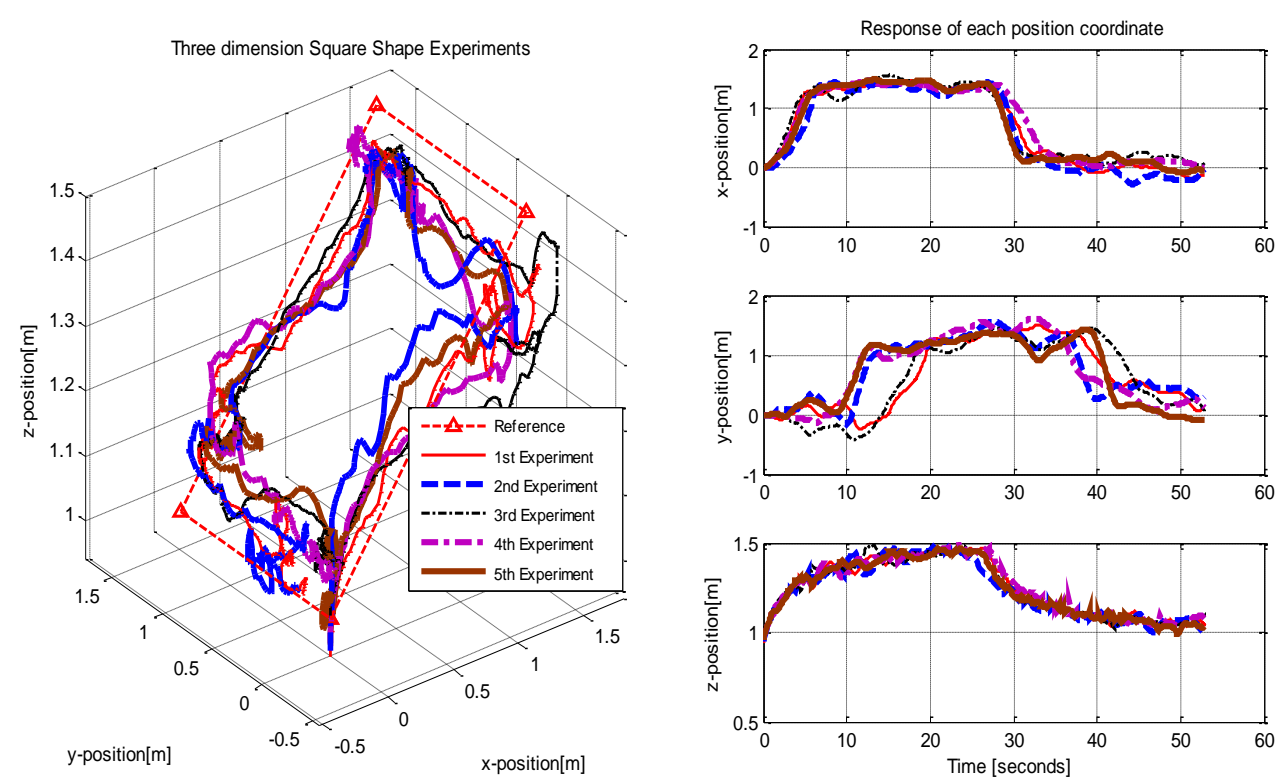

Figure 4. Square shape experiments

The gain scheduling process during the test is shown in Figure 5. It was taken from of one of the experiments above. It is seen that the journey starts from the initial X position error is $1.5 \mathrm{~m}$ so PID control 
used Gain V, while the Y error is zero so the PID control used Gain III and errors Z $0.5 \mathrm{~m}$, PID control used Gain IV. Along with drone journey towards a point of reference, the gain that works is the contribution of the two gain from appropriate membership function. Control signals, in this case, pitch, roll and throttle produced by fuzzy gain scheduling PID control is shown in Figure 6. In these control signals, there are restrictions on the control signal value \pm 0.15 to avoid a collision with the wall due to the cramped indoor space.
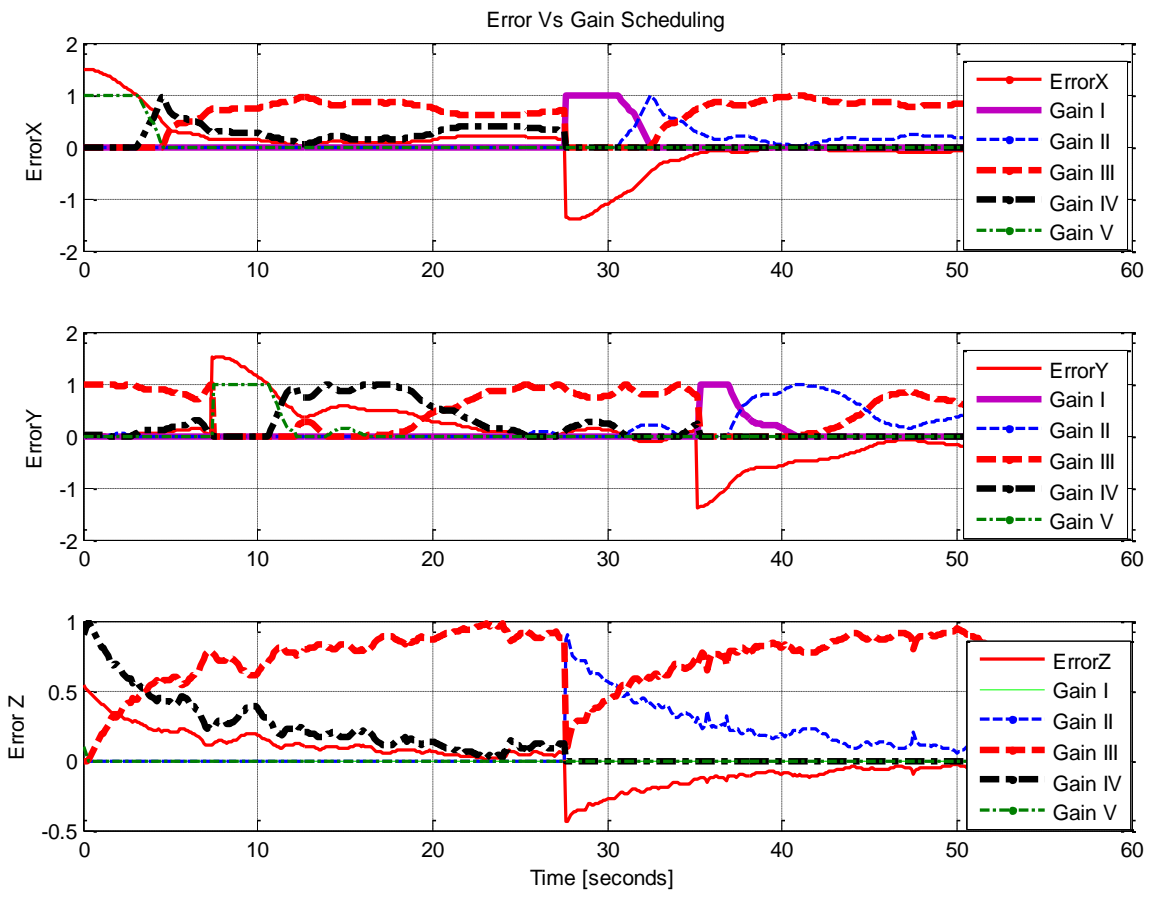

Figure 5. Gain scheduling process
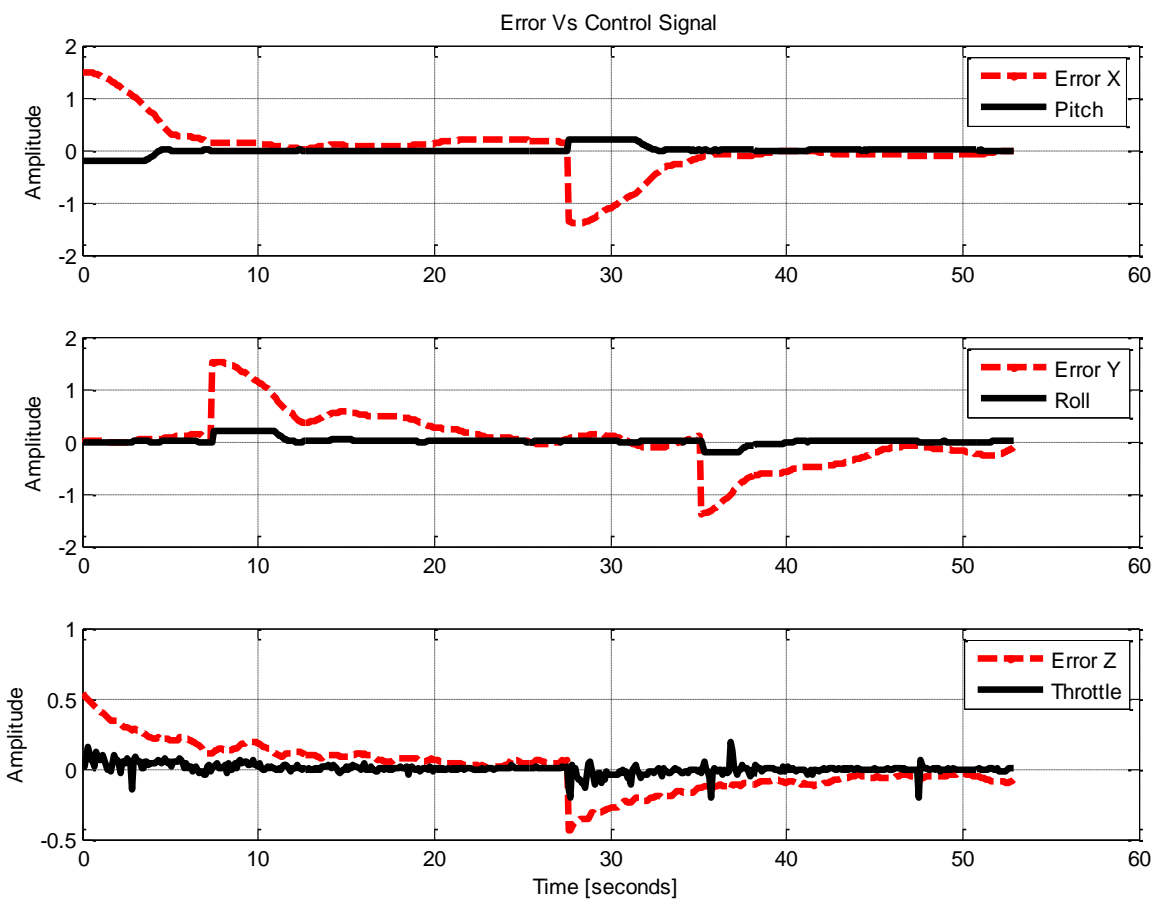

Figure 6. Control signal pitch, roll and throttle 


\section{CONCLUSION}

This paper has implemented fuzzy gain scheduling PID control for position control of AR.Drone. The result of the test, which is to fly to the coordinates $(x, y, z)$ indicates that the PID gain switching process run in accordance with a predetermined operating point. AR.Drone can follow the references given well, but with a small error.

\section{REFERENCES}

[1] P.J. Bristeau, et al., "The Navigation and Control Technology Inside the AR.Drone Micro UAV," 18th IFAC World Congress, 2011.

[2] M. Mogenson, "The AR.Drone LabVIEW Toolkit: A Software Framework for the Control of Low Cost Quadrotor Aerial Robots," Master of Science Thesis, Mechanical Engineering, TUFTS University, 2012.

[3] A. Prayitno, V. Indrawati and G.Utomo, "Trajectory Tracking of AR.Drone Quadrotor Using Fuzzy Logic Controller," Journal Telkomnika, vol. 12, no. 4, pp. 819-828, Dec. 2014.

[4] V. Indrawati, A. Prayitno and T.A. Kusuma, "Waypoint Navigation of AR.Drone Quadrotor Using Fuzzy Logic Controller," Journal Telkomnika,vol. 13, no. 3, pp. 930-939, Sep. 2015.

[5] V. Indrawati, et al., "Comparison of Two Fuzzy Logic Controller Schemes for Position Control of AR.Drone,"7th International Conference on Information Technology and Electrical Engineering (ICITEE), 2015.

[6] A. Prayitno, et al., "Comparison of PID and Fuzzy Controller for Position Control of AR.Drone," IOP Conf. Series: Material Science and Engineering, vol. 190, 2017.

[7] S.Y. Tang, "Vision-Based Control for Autonomous Quadrotor," Final Report :Undergraduated Senior Thesis, Department of Mechanical and Aerospace Engineering, Princeton University, 2013.

[8] R. Abbas and Q. Wu, "Improved Leader Follower Formation Control for Multiple Quadrotors Based AFSA," Journal Telkomnika, vol. 13, no. 1, pp. 85-92, Mar. 2015.

[9] E.A. Seidabad, S.Vandaki and A.V. Kamyad, "Designing Fuzzy PID Controller for Quadrotor," International Journal of Advanced Research in Computer Science \& Technology (IJARCST), vol. 2, no. 4, pp. 221-227, 2014.

[10] D. Gautam and C. Ha, "Control of a Quadrotor Using a Smart Self-Tuning Fuzzy PID Controller," International Journal of AdVanced Robotic Systems, vol. 10, pp. 1-9, 2013.

[11] H. Nasution, "Development of fuzzy logic control for vehicle air conditioning system", TELKOMNIKA (Telecommunication Computing Electronics and Control), 2008, vol. 6, no. 2, pp. 73-82

[12] A. Prayitno, V. Indrawati and C. Arron, "H-Infinity Control for Pitch-Roll AR.Drone," TELKOMNIKA (Telecommunication Computing Electronics and Control), vol. 14, no. 3, pp. 963-973, 2016.

[13] A. Hazzabi, A. Laoufi, I.K. Bousserhane and M. Rahli, "Real Time Implementation of Fuzzy Gain Scheduling of PI Controller for Induction Machine Control," International Journal of Applied Engineering Research., vol. 1, no. 1, pp. 51-60, 2006.

[14] F.U. Syed, et al., "Fuzzy Gain-Scheduling Proportional-Integral Control for Improving Engine Power and Speed Behavior in a Hybrid Electric Vehicle," IEEE Transactions on Vehicular Technology, vol. 58, 2009. 